\title{
Is bronchiectasis an important co-morbidity in COPD?
}

\author{
Dr Timothy Gatheral MRCP PhD, Consultant Respiratory Physician
}

Non cystic-fibrosis bronchiectasis (NCFB) is defined by the British Thoracic Society as 'symptoms of persistent or recurrent bronchial sepsis related to irreversibly damaged and dilated bronchi'. ${ }^{1}$ Typically patients complain of a chronic productive cough and repeated exacerbations associated with increased volume and purulence of sputum, haemoptysis, pleuritic pain, fever and breathlessness. Up to $50 \%$ of cases may be deemed idiopathic. Common established causes include post-infective e.g. tuberculosis, pertussis, measles, related to immune deficiency e.g. hypogammaglobulinaemia, allergic bronchopulmonary aspergillosis (ABPA), and connective tissue disease. Severe NCFB has been considered to be on the retreat in developed countries due to the relative rarity of severe childhood respiratory disease including tuberculosis. It is however clear that NCFB is increasing in prevalence in the United Kingdom (UK) and is a significant cause of respiratory morbidity and mortality. ${ }^{2}$ This is consistent with epidemiologic data from Germany and the United States. ${ }^{3,4}$

The role of bronchiectasis in Chronic Obstructive Pulmonary Disease (COPD) is more controversial. In the first instance it is likely that a significant proportion of bronchiectasis is misdiagnosed as COPD or asthma in the community. These conditions share common symptoms of cough, sputum and breathlessness and may all reveal airflow obstruction on spirometry. The treatment strategies for COPD and NCFB have important differences with much more emphasis on airway clearance physiotherapy, mucolytics and antibiotic therapy in the latter. ${ }^{1}$ Correct identification of NCFB as an alternative or codiagnosis in COPD could therefore be very important to improve respiratory health in the population.

Several studies have indeed now identified a significant prevalence of NCFB in cohorts of patients with COPD. In patients presenting to secondary care with COPD, the prevalence of NCFB has been shown to vary from 20 to $56 \%$ in several studies. A small study in primary care suggested that up to $40 \%$ of patients with coded COPD had evidence of bronchiectasis on high resolution CT (HRCT) scan. ${ }^{5}$ In contrast the ECLIPSE study (a prospective COPD database) suggested a NCFB prevalence of only $4 \%$ on CT chest. $^{6}$

Overall such studies demonstrate that dilated airways are relatively common in COPD. It is less clear whether this is of clinical significance. For example O'Brien's paper $^{5}$ uses CT criteria to diagnose NCFB but this does not take into account relevant symptoms as described in the BTS definition of NCFB. Consequently, several investigators have attempted to determine the clinical impact of $\mathrm{NCFB}$ in COPD. Martinez et al investigated the clinical impact of bronchiectasis on a wellestablished cohort of predominantly male COPD patients in Spain. Patients with concomitant NCFB were more likely to produce purulent sputum, isolate potentially pathogenic organisms, have increased airway inflammation and have a greater number of admissions to hospital with exacerbation. ${ }^{7}$ Conversely Patel et al did not find an association between concomitant NCFB and an increased rate of admission in a UK cohort. ${ }^{8}$

A valid criticism of the true significance of NCFB in COPD has been the ability to differentiate its effects from other markers of severity including airflow obstruction, degree of emphysema and relevant co-morbidities. Bafadhel et al studied HRCT as a tool to phenotype COPD. ${ }^{9}$ In this study NCFB did not independently predict airway inflammation or exacerbation frequency independently of emphysema and bronchial wall thickening observed on the CT scans. Conversely Parr and colleagues did identify a subset of patients with significant NCFB with only mild emphysema and worse health status. ${ }^{10}$ In a prospective study by Martinez et al, NCFB was found to be associated with an increased rate of exacerbation and higher all-cause mortality. ${ }^{11}$ On multivariate analysis this was found to be independent of Forced Expiratory Volume in 1 second (FEV1), Charlson index (a measure of co-morbidity), oxygen status, body mass index, presence of potentially pathogenic microorganisms in sputum, presence of daily sputum production, number of severe exacerbations, and peripheral albumin and ultrasensitive C-reactive protein concentrations.

In support of this research we conducted a retrospective study of patients attending a South London Teaching

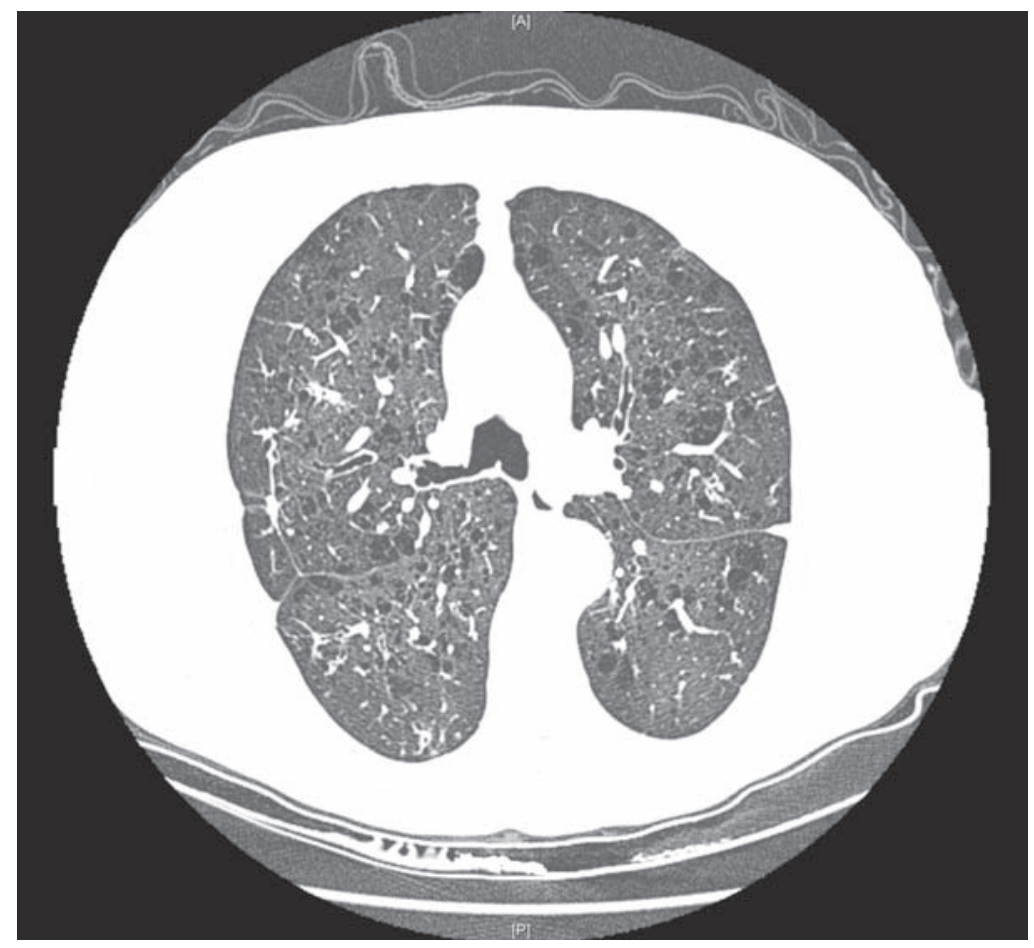

HRCT chest showing combined changes of emphysema, bronchial wall thickening and bronchiectasis 
Hospital with a coded exacerbation of COPD over a 10 year period. ${ }^{12}$ Records were examined for the presence of NCFB and its influence on mortality, exacerbation frequency, and other markers of airway disease including bacterial isolation in sputum. The impact of NCFB was assessed independently of increasing severity emphysema and bronchial wall thickening, age at study entry, gender and Charlson index. Our results suggested that NCFB did not influence mortality in this COPD cohort unlike the data from Martinez et al. ${ }^{11}$ However NCFB was associated with increased hospitalisation and presence of bacterial pathogens on sputum, independent of the degree of emphysema and other aforementioned variables.

So it seems reasonable to suggest that the presence of NCFB influences the clinical phenotype of COPD. A recent meta-analysis comments on trial heterogeneity but reliable signals of increased isolation of potentially pathogenic organisms (especially Pseudomonas aeruginosa), increased hospital stay and a suggestion of increased mortality. ${ }^{13}$ Moreover a very recent paper by Navaratnam et al suggests additional risk of cardiovascular comorbidity in bronchiectasis patients reinforcing the need to take this condition seriously. ${ }^{14}$ However there must be ongoing caution in the absence of a clear causal and mechanistic link between COPD and NCFB. An important recent study may lead to the suggestion that the diagnosis of NCFB in COPD may be misleading. ${ }^{15}$ This relates to the use of the bronchial wall to vessel ratio as the means of reporting the presence of bronchiectasis in HRCT. Pulmonary hypertension is clearly associated with poor outcomes in COPD and results in vessel pruning on CT. It is possible that reported NCFB on patient scans does not reflect true bronchial dilatation at all but rather the vasculopathy of pulmonary hypertension. It will be crucial in future studies that the presence of NCFB in concomitant COPD is backed up by relevant clinical symptoms including sputum production and infective exacerbations.

In summary there is considerable, although still controversial, evidence that NCFB is associated with worse health outcomes in COPD. Given the significant differences in management between COPD and NCFB more research is needed to examine the effects of improved diagnosis of NCFB and targeted management on clinical outcomes in this population. This could include screening trials in primary care and the use of structured integrated care pathways to improve the management of patients with bronchiectasis.

\section{REFERENCES}

1. Pasteur M.C., Bilton, D. \& Hill, A.T. (2010) British Thoracic Society guideline for non-CF bronchiectasis, Thorax, 65 Suppl 1, pp. i1-58.

2. Quint, J.K., Millett, E.R., Joshi, M., Navaratnam, V., Thomas, S.L., Hurst, J.R., Smeeth, L. \& Brown, J.S. (2016) Changes in the incidence, prevalence and mortality of bronchiectasis in the UK from 2004 to 2013: a population-based cohort study, Eur Respir F, 47(1), pp. 186-93.

3. Ringshausen, F.C., de Roux, A., Pletz, M.W., Hämäläinen, N., Welte, T. \& Rademacher, J. (2013) Bronchiectasis-associated hospitalizations in Germany, 2005-2011: a population-based study of disease burden and trends, PLoS One, 1;8(8), e71109.
4. Seitz, A.E., Olivier, K.N., Adjemian, J., Holland, S.M. \& Prevots, D.R. (2012) Trends in bronchiectasis among Medicare beneficiaries in the United States, 2000-2007, Chest, 142(2), pp. 432-9.

5. O'Brien, C., Guest, P.J., Hill, S.L. \& Stockley, R.A. (2000) Physiological and radiological characterisation of patients diagnosed with chronic obstructive pulmonary disease in primary care, Thorax, 55(8), pp. 635-642.

6. Agusti, A., Calverley, P.M., Celli, B., Coxson, H.O., Edwards, L.D. \& Lomas, D.A. et al. (2010) Characterisation of COPD heterogeneity in the ECLIPSE cohort, Respir Res, 11:122.

7. Martinez-Garcia, M.A., Soler-Cataluna, J.J., Donat Sanz, Y., Catalan, Serra P., Agramunt, Lerma M. \& Ballestin, Vicente. J. et al. (2011) Factors associated with bronchiectasis in patients with COPD, Chest, 140(5), pp. 1130-1137.

8. Patel, I.S., Vlahos, I., Wilkinson, T.M., Lloyd-Owen, S.J., Donaldson, G.C. \& Wilks M., et al. (2004) Bronchiectasis, exacerbation indices, and inflammation in chronic obstructive pulmonary disease, Am 7 Respir Crit Care Med, 170(4), pp. 400-407.

9. Bafadhel, M., Umar, I., Gupta, S., Raj, J.V., Vara, D.D. \& Entwisle, J.J. et al. (2011) The role of CT scanning in multidimensional phenotyping of COPD, Chest, 140(3), pp. 634-642.

10. Parr, D.G., Guest, P.G., Reynolds, J.H., Dowson, L.J.\& Stockley, R.A. (2007) Prevalence and impact of bronchiectasis in alpha1-antitrypsin deficiency, $A m \mathcal{F}$ Respir Crit Care Med, 176(12), pp. 1215-1221.

11. Martinez-Garcia, M.A., de la Rosa, Carrillo D., SolerCataluna, J.J., Donat-Sanz, Y., Serra, P.C. \& Lerma, M.A., et al. (2013) Prognostic value of bronchiectasis in patients with moderate-to-severe chronic obstructive pulmonary disease, Am 7 Respir Crit Care Med, 187(8), pp. 823-831.

12. Gatheral, T., Kumar, N., Sansom, B., Lai, D., Nair, A., Vlahos, I. \& Baker, E.H. (2014) COPD-related Bronchiectasis; Independent Impact on Disease Course and Outcomes, COPD, 11(6) pp. 605-14.

13. Du, Q., Jin, J., Liu, X. \& Sun Y. (2016) Bronchiectasis as a comorbidity of chronic obstructive pulmonary disease: A systematic review and meta-analysis, $P L O S$ ONE, 11(3): 0150532.

14. Navaratnam, V., Millett, E.R., Hurst, J.R., Thomas, S.L., Smeeth, L., Hubbard, R.B., Brown, J. \& Quint, J.K. (2017) Bronchiectasis and the risk of cardiovascular disease: a population-based study, Thorax, 72(2), pp. 161-166.

15. Diaz, A.A., Young, T.P., Maselli, D.J., Martinez, C.H., Gill, R., Nardelli, P., Wang, W., Kinney, G.L., Hokanson, J.E., Washko, G.R. \& San Jose Estepar, R. (2016) Quantitative CT measures of bronchiectasis in smokers, Chest. 24. pii: S00123692(16)62460-1.

Correspondence to: tim.gatheral@mbht.nhs.uk 